\title{
Perfil empreendedor entre estudantes de graduação em enfermagem
}

\author{
Entrepreneurial profile among undergraduate nursing students
} Perfil emprendedor de estudiantes universitarios de enfermería

\section{Alisson Jofre ${ }^{1}$ (B) https://orcid.org/0000-0002-6351-0150 \\ Kelli Pazeto Della Giustina ${ }^{1}$ ๗ic https://orcid.org/0000-0001-9262-0116 \\ Greice Lessa ${ }^{1}$ ic https://orcid.org/0000-0001-7056-3317}

Marilene Nonnemacher Luchtemberg ${ }^{2}$ ib hittps://orid. org/0000-0002-9728-9634

Bruno de Campos Gobato ${ }^{3}$ id https://orcid.org/0000-0002-6861-5146

João Lucas Campos de Oliveira ${ }^{4}$ iD https://orcid.org/0000-0002-1822-2360

Alacoque Lorenzini Erdmann ${ }^{3}$ io https://orcid.org/0000-0003-4845-8515

José Luís Guedes dos Santos ${ }^{3}$ io https://orcid.org/0000-0003-4845-8515

Como citar:

Jofre A, Giustina KP, Lessa G, Luchtemberg

MN, Gobato BC, Oliveira JL, et al. Perfil

empreendedor entre estudantes de graduação em enfermagem. Acta Paul Enferm. 2021;34:eAPE001645.

DOI

http://dx.doi.org/10.37689/actaape/2021A0001645

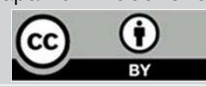

Descritores

Mercado de trabalho; Contrato de risco; Estudantes de enfermagem; Educação em enfermagem

Keywords

Job market; Entrepreneurship; Students, nursing;

Education nursing

Descriptores

Mercado de trabajo; Contrato de riesgo; Estudiantes de enfermería; Educación en

enfermería

Submetido 29 de Junho de 2020

Aceito

7 de Dezembro de 2020

Autor correspondente José Luís Guedes dos Santos E-mail: jose.santos@ufsc.br

\section{Resumo}

Objetivo: Identificar o perfil empreendedor entre estudantes de graduação em enfermagem.

Métodos: Estudo transversal, desenvolvido em 2018, com 239 estudantes de enfermagem de duas Instituições de Ensino Superior de Santa Catarina, Brasil. A coleta de dados ocorreu por meio de questionário de caracterização pessoal e instrumento validado sobre avaliação do perfil empreendedor no meio acadêmico. Utilizou-se estatística descritiva para análise dos dados.

Resultados: Predominaram estudantes na faixa etária de até 20 anos (38,9\%), do sexo feminino $(88,1 \%)$ e dos dois primeiros anos do curso (51\%). Em relação ao perfil empreendedor, 155 (64,9\%) alunos foram classificados no nível superior e 84 (35,1\%) no nível médio superior. 0 domínio "Energia e Comprometimento" obteve a melhor pontuação $(4,49 \pm 0,57)$ e "Capacidade de Assumir Riscos Moderados" a média mais baixa $(3,71 \pm 0,56)$, mas ainda acima da mediana da escala.

Conclusão: Há concentração de níveis elevados de empreendedorismo no perfil de estudantes de graduação em enfermagem.

\section{Abstract}

Objective: To identify the entrepreneurial profile among undergraduate nursing students.

Methods: Cross-sectional study, developed in 2018, with a total of 239 nursing students from two Higher Education Institutions in Santa Catarina, Brazil. Data collection took place through a personal characterization questionnaire and a validated instrument on the evaluation of the entrepreneurial profile in the academic environment. Descriptive statistics were used for data analysis.

Results: There was a predominance of students aged up to 20 years old (38.9\%), female (88.1\%) and the first two years of the course (51\%). Regarding the entrepreneurial profile, $155(64.9 \%)$ students were classified at the higher level and $84(35.1 \%)$ at the upper medium level. The domain "Energy and Commitment" obtained the best score $(4.49 \pm 0.57)$ and "Ability to Take Moderate Risks", the lowest average $(3.71 \pm 0.56)$, but still above the median of the scale.

Conclusion: There is a concentration of high levels of entrepreneurship in the profile of undergraduate nursing students.

\section{Resumen}

Objetivo: Identificar el perfil emprendedor de estudiantes universitarios de enfermería. 
Métodos: Estudio transversal, llevado a cabo en 2018 con 239 estudiantes de enfermería de dos instituciones de educación superior del estado de Santa Catarina, Brasil. La recopilación de datos se realizó mediante un cuestionario de caracterización personal y un instrumento validado sobre evaluación del perfil emprendedor en el medio académico. Se utilizó la estadística descriptiva para el análisis de los datos.

Resultados: Predominaron estudiantes del grupo de edad hasta 20 años (38,9\%), de sexo femenino (88,1\%) y de los dos primeros años de la carrera (51 \%). Con relación al perfil emprendedor, 155 alumnos (64,9 \%) fueron clasificados en el nivel superior y $84(35,1 \%)$ en el nivel intermedio superior. El dominio "Energía y compromiso" obtuvo la mejor puntuación $(4,49 \pm 0,57)$ y "Capacidad de asumir riesgos moderados" el promedio más bajo $(3,71 \pm 0,56)$, pero aún por arriba de la mediana de la escala.

Conclusión: Existe una concentración de niveles elevados de una tendencia emprendedora en el perfil de estudiantes universitarios de enfermería.

\section{Introdução}

A globalização, o desenvolvimento de novas tecnologias e as transformaçóes sociais têm demandado a formação de profissionais com visão sistêmica, capazes de acompanhar essa dinamicidade. $\mathrm{Na}$ área da saúde, vislumbra-se cada vez mais a necessidade de os enfermeiros estarem atualizados frente às mudanças, o que impóe o desenvolvimento de competências para inserção no mercado de trabalho e atendimento das necessidades da sociedade. ${ }^{(1)}$ $\mathrm{O}$ aprimoramento profissional do enfermeiro deve acontecer desde a graduação visando, inclusive, a formação de um perfil empreendedor. Esse perfil compóe espírito crítico, proatividade, criatividade, disposição para a inovaçáo, atitude positiva e capacidade de negociação, logo, habilidade de aprender a conhecer, aprender a fazer, a conviver e a ser. ${ }^{(2,3)}$

O empreendedorismo é conceituado, de modo geral, como o ato de fazer algo novo e diferente. Isso pode ocorrer dentro de um mercado (empreendedorismo empresarial), de uma empresa ou organização (intraeempreendedorismo) ou para a sociedade (empreendedorismo social). ${ }^{(4)} \mathrm{Na}$ Enfermagem, o empreendedorismo social ocorre quando o enfermeiro atua como agente de mudanças e transformaçóes na situação de saúde de pacientes e famílias inseridos na comunidade. $\mathrm{O}$ intraempreendedorismo remete à atuaçáo de enfermeiros como agentes de mudança e inovação em organizaçóes públicas e privadas, nas quais atuam como empregados. O empreendedorismo empresarial caracteriza-se pela prática autônoma de enfermeiros, como, por exemplo, em consultórios no atendimento de pacientes com feridas, cuidado domiciliar, assistência privada nos serviços de obstetrícia e puerpério materno. ${ }^{(5)}$

Assim, diante dessas tipologias, entende-se que o empreendedorismo não é apenas uma competência importante para a busca da prática autônoma, mas uma característica que potencializa a atuação do enfermeiro na gestáo e no cuidado às pessoas e coletividades. ${ }^{(5)}$ Por meio do empreendedorismo, os enfermeiros podem, por exemplo, desenvolver inovaçóes no processo de cuidar em saúde, contribuindo para o reconhecimento e visibilidade social do trabalho da enfermagem. ${ }^{(6)}$

Nesse sentido, pontua-se a necessidade de investimento na produção científica sobre empreendedorismo na enfermagem, especialmente quanto à formação empreendedora do estudante de enfermagem. ${ }^{(4,6)}$ Essa foi uma lacuna evidenciada em uma revisão de literatura publicada em 2019 sobre empreendedorismo na enfermagem, que incluiu bases de dados internacionais como Publisher Medline (PUBMED), SciVerse Scopus (SCOPUS), e Cumulative Index to Nursing and Allied Health Literature (CINAHL). ${ }^{(4)}$

A formação de enfermeiros com potencial transformador em nível social, organizacional e político requer a presença de competências empreendedoras em seu perfil acadêmico. Por esse motivo, estudos sobre o perfil empreendedor de estudantes ao longo da formação em enfermagem são importantes para alavancar a visão sobre empreendedorismo na profissão e potencializar a busca por autonomia e qualificação profissional. Além disso, pesquisa sobre essa temática também contribuem para o avanço e a consolidação da Ciência da Enfermagem. Ante o exposto, questionou-se: qual é o perfil empreendedor de estudantes de graduação em enfermagem?

Desse modo, o objetivo deste estudo foi identificar o perfil empreendedor entre estudantes de graduação enfermagem.

\section{Métodos}

Trata-se de um estudo observacional descritivo. Foi desenvolvido em duas Instituiçôes de Ensino 
Superior (IES) privadas, localizadas no interior do estado de Santa Catarina, Brasil. Os cursos de enfermagem de ambas possuem cinco anos de duração e são desenvolvidos no período noturno. Apresentam objetivo e organização pedagógica semelhantes, com foco na formação de enfermeiros generalistas com competências técnicas e científicas para atuarem de forma reflexiva, crítica, criativa, humanista e transformadora. Nenhum dos cursos inclui disciplinas específicas sobre empreendedorismo na grade curricular. Porém, o tema é abordado em disciplinas relacionas ao exercício profissional, mercado de trabalho e/ou gerenciamentos dos serviços de saúde e enfermagem.

A população do estudo foi constituída pelos 288 discentes matriculados nos cursos de graduação em Enfermagem das IES. A amostragem foi não probabilística e por conveniência, pois previa a participação da população total. $\mathrm{O}$ critério de inclusão foi estar regularmente matriculado no Curso de Enfermagem. O critério de exclusão foi estar ausente no momento da coleta de dados, devido a atestado médico ou outro afastamento de qualquer natureza. Ao total, 47 participantes foram excluídos por motivo de ausência. Também foram excluídos dois alunos que estavam participando do projeto de pesquisa. Assim, a amostra foi composta por 239 alunos, correspondendo a $82,9 \%$ da população.

A coleta de dados ocorreu durante o mês de agosto de 2018, por meio de um questionário autoaplicável composto por questóes de caracterização pessoal (idade, sexo e ano do curso) e o instrumento de Avaliaçáo de Perfil Empreendedor em Meio Acadêmico, o qual foi adaptado de formulários elaborados pelo Serviço Brasileiro de Apoio às Micro e Pequenas Empresas (SEBRAE) e validado para uso entre estudantes universitários. ${ }^{(7)}$ Os participantes da pesquisa foram abordados pessoalmente pela equipe de pesquisa, durante o horário das aulas e mediante concordância da coordenação do curso e do professor responsável pela disciplina.

$\mathrm{O}$ instrumento de Avaliação de Perfil Empreendedor em Meio Acadêmico é composto por 40 questóes respondidas pelos estudantes a partir de uma escala Likert com valores de 1 a 5, variando de " $1=$ Discordo Totalmente" a " $5=$ Concordo
Totalmente". As questôes são distribuídas em oito domínios/dimensóes: Autonomia e Confiança; Busca de Oportunidades e Visão; Capacidade de Assumir Riscos Moderados; Capacidade de Inovar; Energia e Comprometimento; Liderança e Necessidade de Poder; Obstinação e Necessidade de Realização; e Planejamento Sistemático. ${ }^{(7)}$

A partir da soma de cada item, encontra-se o perfil empreendedor do respondente. Quanto mais próxima a somatória estiver de 200 (escore máximo), maior é a presença de atributos empreendedores. Escores maiores que 160 representam perfil empreendedor superior. De 121 a 160 pontos, o perfil é considerado médio superior. Entre 81 e 120 pontos, o perfil é médio. De 41 a 80 pontos, considera-se perfil médio inferior. Por fim, resultados abaixo de 40 pontos indicam um perfil empreendedor inferior. ${ }^{(7)}$

Os dados foram coletados manualmente e transpostos em uma planilha no software Excel ${ }^{\circledR}$. A análise dos dados foi realizada por meio de estatística descritiva no software Statistical Package for Social Sciences (SPSS), versão 20.0. As variáveis categóricas foram apresentadas na forma de frequências absolutas (n), relativas (\%), e Intervalo de Confiança 95\% para proporções. A variável quantitativa idade foi categorizada em faixas etárias. As variáveis intervalares da escala foram expressas por medidas de tendência central (média e mediana) e dispersão (desvio padrão, mínimo e máximo).

Os aspectos éticos foram respeitados, conforme Resolução no 466/2012 do Conselho Nacional de Saúde. O projeto foi aprovado pelo Comitê de Ética de referência, com parecer $\mathrm{n}^{\circ} 2.876 .878$ e CAAE: 93974118.0.0000.5598.

\section{Resultados}

Do total de 239 participantes do estudo, a maioria era da faixa etária de até 20 anos ( $\mathrm{n}=93 ; 38,9 \%)$ e do sexo feminino ( $\mathrm{n}=208 ; 88,1 \%)$. Quanto ao ano do curso, pouco mais da metade $(\mathrm{n}=122 ; 51,0 \%)$ dos alunos estavam matriculados em séries iniciais do curso, ou seja, no $1^{\circ}(\mathrm{n}=61 ; 25,5 \%)$ e $2^{\circ}$ anos $(\mathrm{n}=61 ; 25,5 \%)$ (Tabela 1$)$. 
Tabela 1. Caracterização demográfica e ano do curso de graduação dos estudantes de enfermagem

\begin{tabular}{|c|c|c|}
\hline Variável & $n(\%)$ & IC $95 \%{ }^{*}$ \\
\hline \multicolumn{3}{|l|}{ Faixa etária ( $n=239$ ) } \\
\hline Até 20 anos & $93(38,9)$ & $32,7-45,4$ \\
\hline 21 a 25 anos & $85(35,6)$ & $29,5-42,0$ \\
\hline 26 a 30 anos & $23(9,6)$ & $6,2-14,1$ \\
\hline 31 a 35 anos & $20(8,4)$ & $5,2-12,6$ \\
\hline Mais de 35 anos & $18(7,5)$ & $4,5-11,6$ \\
\hline \multicolumn{3}{|l|}{$\operatorname{Sexo}(n=236)^{\star \star}$} \\
\hline Feminino & $208(88,1)$ & $83,3-92,0$ \\
\hline Masculino & $28(11,9)$ & $8,0-16,7$ \\
\hline \multicolumn{3}{|c|}{ Ano do Curso ( $\mathrm{n}=239$ ) } \\
\hline $1^{0}$ & $61(25,5)$ & $20,1-31,5$ \\
\hline $2^{0}$ & $61(25,5)$ & $20,1-31,5$ \\
\hline $3^{\circ}$ & $44(18,4)$ & $13,7-23,9$ \\
\hline $4^{\circ}$ & $33(13,8)$ & $9,7-18,8$ \\
\hline $5^{0}$ & $40(16,8)$ & $20,1-31,5$ \\
\hline
\end{tabular}

*Intervalo de Confiança $95 \%$ para proporções. *^ Número inferior ao total da amostra, pois três participantes não responderam essa questão

Em relação ao perfil empreendedor, 155 (64,9\%) alunos ficaram classificados no nível superior e 84 $(35,1 \%)$ no nível médio superior. Nenhum aluno obteve pontuação referente aos níveis inferior, médio inferior e médio. Entre os alunos classificados no nível superior, predominaram os do sexo masculino $(71,4 \%)$, no $3^{\circ}$ ano do curso $(68,2 \%)$ e na faixa etária de 30 a 35 anos (80\%) (Tabela 2).

Tabela 2. Perfil empreendedor superior e médio superior conforme sexo, ano do curso e faixa etária dos estudantes de enfermagem

\begin{tabular}{lcc}
\hline Variável & $\begin{array}{c}\text { Superior } \\
\mathbf{n}(\%)\end{array}$ & $\begin{array}{c}\text { Médio Superior } \\
\mathbf{n}(\%)\end{array}$ \\
\hline $\begin{array}{l}\text { Sexo }(\mathrm{n}=236) \\
\text { Masculino }\end{array}$ & \\
$\quad$ Feminino & $20(71,4)$ & $8(28,6)$ \\
Anos do Curso $(\mathrm{n}=239)$ & $132(63,5)$ & $76(36,5)$ \\
$1^{\circ}$ & & \\
$2^{0}$ & $40(65,6)$ & $21(34,4)$ \\
$3^{\circ}$ & $39(63,9)$ & $22(36,1)$ \\
$4^{0}$ & $30(68,2)$ & $14(31,8)$ \\
$5^{\circ}$ & $21(63,6)$ & $12(36,4)$ \\
Faixa etária (n=239) & $25(62,5)$ & $15(37,5)$ \\
Até 20 anos & & \\
21 a 25 anos & $54(58,1)$ & $39(41,9)$ \\
26 a 30 anos & $55(64,7)$ & $30(35,3)$ \\
31 a 35 anos & $16(69,6)$ & $7(30,4)$ \\
Mais de 35 anos & $16(80)$ & $4(20)$ \\
\hline & $14(77,8)$ & $4(22,2)$ \\
\hline
\end{tabular}

$\mathrm{Na}$ análise das dimensôes do perfil empreendedor, a dimensão "Energia e Comprometimento" obteve a maior/melhor pontuação. O pior escore foi identificado na dimensão "Capacidade de Assumir Riscos Moderados” (Tabela 3).
Tabela 3. Dimensões de análise do perfil empreendedor de estudantes de enfermagem $(n=239)$

\begin{tabular}{|c|c|c|c|c|c|}
\hline Dimensões & Média & Mediana & $\mathrm{DP}^{*}$ & Mínimo & Máximo \\
\hline Autonomia e Confiança & 4,07 & 4,14 & 0,52 & 2,43 & 5,00 \\
\hline $\begin{array}{l}\text { Busca de Oportunidades } \\
\text { e Visão }\end{array}$ & 4,29 & 4,33 & 0,69 & 1,33 & 6,00 \\
\hline $\begin{array}{l}\text { Capacidade de Assumir } \\
\text { Riscos Moderados }\end{array}$ & 3,71 & 3,71 & 0,56 & 2,00 & 5,00 \\
\hline Capacidade de Inovar & 4,29 & 4,33 & 0,62 & 2,33 & 6,33 \\
\hline Energia e Comprometimento & 4,49 & 4,66 & 0,57 & 2,33 & 5,33 \\
\hline $\begin{array}{l}\text { Liderança e Necessidade } \\
\text { de Poder }\end{array}$ & 3,93 & 4,00 & 0,58 & 2,17 & 5,83 \\
\hline $\begin{array}{l}\text { Obstinação e Necessidade de } \\
\text { Realização }\end{array}$ & 4,39 & 4,40 & 0,51 & 2,80 & 5,60 \\
\hline Planejamento Sistemático & 4,24 & 4,33 & 0,58 & 2,67 & 5,83 \\
\hline
\end{tabular}

A análise do perfil dos participantes mostra que a inserção no ensino superior está ocorrendo cada vez mais cedo, ao menos no contexto investigado, pois a maioria dos acadêmicos tinha até 20 anos $(38,9 \%)$. Esse achado vai ao encontro de estudos anteriores que evidenciaram a predominância de estudantes de enfermagem com até 25 anos. ${ }^{(8-11)}$

Com o objetivo de gerar perspectivas de crescimento e progresso, a entrada de jovens no ensino superior pode ser considerada como um fator positivo, refletindo uma melhora no acesso à formação universitária. Em contrapartida, a opção precoce pela profissão demanda do estudante amadurecimento no que se refere à certeza da escolha pela carreira. Nesse sentido, a inexperiência pessoal pode dificultar o enfrentamento das reais condiçôes de trabalho da enfermagem e comprometer o desenvolvimento de competências relacionadas ao empreendedorismo. ${ }^{(12)}$

Quanto ao sexo, a predominância de mulheres nos cursos de enfermagem já é uma evidência amplamente descrita da literatura. ${ }^{(7,10,11,13)}$ Além disso, reflete uma característica histórica da Enfermagem em âmbito global que perdura até os dias atuais, embora se observe gradativamente maior inserção de homens na profissão. ${ }^{(11,14)}$

No tangente ao perfil empreendedor, os resultados obtidos foram positivos na medida em que a maioria dos alunos ficou classificada no nível superior $(64,9 \%)$ ou médio superior $(35,1 \%)$. Não foram identificados estudos na Enfermagem utilizando o mesmo instrumento a fim de comparar 
os índices obtidos. Porém, os achados deste estudo assemelham-se aos resultados de pesquisas com alunos formandos do curso de Comércio Internacional de uma universidade do Rio Grande do $\mathrm{Sul}^{7}$ e estudantes de Odontologia de uma universidade pública de Santa Catarina. ${ }^{(15)}$

Estudos na Enfermagem usando o teste de Tendência Empreendedora Geral (TEG) encontraram resultados diferentes aos descritos na pesquisa ora apresentada. Segundo esse instrumento, as características empreendedoras dos enfermeiros ficaram abaixo da média geral. ${ }^{(16,17)}$ Apesar de serem instrumentos distintos, um aspecto semelhante do TEG com o instrumento utilizado neste estudo é que ambos possuem domínio acerca da propensão à riscos, dito como uma característica do empreendedorismo; e que, de forma correlata a esta pesquisa, foi o pior domínio avaliado também entre residentes de enfermagem. ${ }^{(17)}$ Isso pode estar relacionado à característica predominantemente institucional da Enfermagem, a qual, de certa forma, contribui para que o profissional priorize a inserção como trabalhador em uma organização ou serviço de saúde, em detrimento, por exemplo, da busca por uma prática autônoma como empreendedor.

O empreendedorismo é uma competência que pode ser influenciada por características psicológicas, contexto social, antecedentes culturais e educacionais, sendo difícil analisar essas influências em uma única pesquisa. Desse modo, o fato de nesta pesquisa nenhum acadêmico ter obtido um escore baixo para o perfil empreendedor indica um preparo promissor na carreira profissional em enfermagem. Em contrapartida, é prudente assumir que o fato de características altamente positivas terem sido expressas na autopercepção dos estudantes não garante comportamentos empreendedores no futuro, até mesmo porque, como já enunciado, a competência tende a ser influenciada por múltiplos fatores, incluindo o contexto social e a dinâmica de mercado.

Estudantes do sexo masculino evidenciaram maior concentração do perfil empreendedor superior $(71,4 \%)$ em comparação ao sexo feminino nesse mesmo enquadramento $(63,5 \%)$. Estudo sobre características empreendedoras de estudantes de enfermagem na Turquia também evidenciou maior inclinação de alunos do sexo masculino para o empreendedorismo. A pesquisa considera que tal achado se relaciona à menor propensão das mulheres em assumir riscos, em decorrência de um contexto cultural e socioeconômico que estimula a autoconfiança masculina e reforça o papel materno e as responsabilidades domésticas das mulheres. ${ }^{(18)}$ Logo, reafirma-se a influência social dessa competência, até mesmo de valores com potencial estigmatizante.

Nesse sentido, vale pontuar também os achados de estudo sobre os desafios ao desenvolvimento de ações empreendedoras por enfermeiras em posição de liderança em diferentes organizaçóes e serviços de saúde no Sul do Brasil. Apesar da Enfermagem ser exercida majoritariamente por mulheres, as enfermeiras entrevistadas consideraram que são acometidas por discriminação de gênero e dificuldades na criação e implementação de projetos. Além disso, consideram que sua chefia imediata, em geral, dificulta a mobilização para o empreendedorismo, endossando um comportamento que desacredita a capacidade de gestão das mulheres. ${ }^{(19)}$

Os estudantes do $3^{\circ}$ ano apresentam maior proporção no perfil empreendedor superior $(68,2 \%)$. Esse resultado pode estar associado ao envolvimento dos alunos em atividades teórico-práticas no momento intermediário do curso, o que os aproximam do exercício profissional. Além disso, é um período marcado por preocupações em relação à inserção do mercado de trabalho entre os estudantes, o que pode levá-los a uma dedicação maior no desenvolvimento de características empreendedoras, como liderança, comunicação e tomada de decisão. De forma similar, estudo australiano ressalta a contribuição da inserção do aluno em ambientes clínicos para a obtenção de uma visão mais profunda de seu futuro papel profissional como enfermeiro. ${ }^{(20)}$

A faixa etária com destaque ao perfil empreendedor superior foi a de 31 a 35 anos, em seguida de mais de 36 anos. Esse resultado sugere que pessoas com maior experiência acumulada de vida sobressaem-se quanto às habilidades pessoais e profissionais vinculadas ao empreendedorismo.

Estudo com residentes de enfermagem brasileiros constatou que alunos com mais idade e experiência profissional apresentaram mais impulso e 
determinação. ${ }^{(21)}$ Em contraponto, estudos indicam a importância de traços de personalidade para inclinação empreendedora, sendo que as características empreendedoras predominam entre pessoas mais jovens. ${ }^{(18,22)}$ Assim, quanto mais cedo iniciar o desenvolvimento de uma educação para o empreendedorismo, maior será a autoconfiança, criatividade e potencial de inovação dos alunos.

Entre as dimensóes do perfil empreendedor dos estudantes de enfermagem, o destaque para "Energia e Comprometimento" pode estar associado à satisfação e autoconfiança decorrentes do momento vivido de aprendizagem e desenvolvimento acadêmico. Além disso, esse resultado pode estar relacionado ao investimento crescente dos cursos de enfermagem na utilização de metodologias ativas, que estimulam envolvimento dos alunos no seu processo de ensino. ${ }^{(23)}$

Outro resultado que chama atenção em relação às dimensôes do perfil empreendedor é o escore do domínio "Liderança e Necessidade de Poder", o segundo pior entre os oito domínios avaliados. Estudo realizado na Finlândia obteve resultados semelhantes a partir da autoavaliação de estudantes de enfermagem sobre as competências profissionais desenvolvidas ao longo da graduação. Os estudantes consideram-se mais competentes para a realização de atividades de cuidado direto ao paciente do que para açóes gerenciais, relacionadas à planejamento, liderança e tomada de decisão. ${ }^{(24)}$ Assim, é válido pontuar a importância de discussóes acerca da formação do enfermeiro, no que tange ao enfoque dado ao desenvolvimento de competências e habilidades para a liderança e ao posicionamento político relacionados à dimensão gerencial do trabalho da enfermagem.

É importante ressaltar que, apesar da liderança permear a dimensão gerencial do trabalho do enfermeiro, e, em nível de formação, isso ser vinculado com frequência à área/disciplina de Administração/ Gestão/Gerenciamento em Enfermagem, ${ }^{(25)}$ acredita-se que o desenvolvimento do empreendedorismo de forma geral merece transversalidade na educação do enfermeiro. Isso porque não se remete exclusivamente a aspectos técnico-instrumentais de gerência ou à visão de mundo/mercado/profissão. Os im- pulsos empreendedores não se limitam à gestão do serviço ou negócio, incluem o próprio cuidado de enfermagem em diferentes espaços de produção.

Os resultados do estudo apresentam limitação em relação ao contexto investigado, pois a pesquisa foi desenvolvida somente em instituiçóes de ensino superior por meio de uma amostra por conveniência, sem ponderação de diferenças entre fases e cursos. Além disso, características psicológicas, aspectos culturais e motivação dos participantes influenciam a educação de diferentes maneiras, que não podem ser controladas no momento de uma pesquisa. Também é preciso considerar que o instrumento utilizado para analisar o empreendedorismo não é específico para área de Enfermagem, ou seja, náo contempla a visão ampla do empreendedorismo para a profissão, incluindo o social.

Apesar dessas limitaçóes, considera-se que a investigação trouxe resultados satisfatórios e importantes, já que as instituiçóes de ensino pesquisadas poderão conhecer os resultados obtidos e utilizá-los no aprimoramento de políticas de ensino, pesquisa e extensão com foco no empreendedorismo. Além disso, os achados da pesquisa poderão subsidiar novos estudos visando a avanços teóricos em relação à área investigada. Nesse sentido, ressalta-se a importância de estudos qualitativos ou de metodologia mista para aprofundar e complementar a descrição dos achados deste estudo.

\section{Conclusão}

A pesquisa evidenciou o predomínio de níveis elevados de características empreendedoras no perfil de estudantes de enfermagem, principalmente entre participantes do sexo masculino, no $3^{\circ}$ ano do curso e na faixa etária de 30 a 35 anos. Nenhum acadêmico obteve perfil empreendedor médio ou baixo. Apesar de não garantir práticas empreendedoras no futuro exercício profissional, essa realidade pode fundamentar direcionamentos que potencializem a atuação do enfermeiro como agente transformador estratégico da realidade e suscitar discussóes acerca de sua visibilidade social. 


\section{Agradecimentos}

\section{Ao Conselho Nacional de Desenvolvimento Científico e Tecnológico $(\mathrm{CNPq})$, processo $\mathrm{n}^{\circ}$ 424869/2018-7.}

\section{Colaborações}

\begin{abstract}
Jofre A, Giustina KP, Lessa G, Luchtemberg MN, Gobato BC, Oliveira JLC, Erdmann AL e Santos JLG colaboraram com a concepçáo do estudo, análise e interpretação dos dados, redação do artigo, revisão crítica relevante do conteúdo intelectual e aprovação da versão final a ser publicada.
\end{abstract}

\section{Referências}

1. Backes DS, Haag BK, Vasconcelos J, Dalcin CB, Backes MT, Lomba L. Nursing students in the community: entrepreneurial strategy and proponent of changes. Rev Bras Enferm. 2018;71(4 Suppl 4):1799804.

2. Püschel VA, Costa D, Reis PP, Oliveira LB, Carbogim FD. Nurses in the labor market: professional insertion, competencies and skills. Rev Bras Enferm. 2017;70(6):1220-6.

3. Fernandes JD, Rebouças LC. A decade of national curriculum guidelines for Graduation in Nursing: advances and challenges. Rev Bras Enferm 2013;66(Spe):95-101.

4. Copelli FH, Erdmann AL, Santos JL, Lanzoni GM, Andrade SR Entrepreneurship in the public university management of nursing: obstacles and strategies. Rev Rene. 2017;18(5):577-83.

5. Copelli FH, Erdmann AL, Santos JL. Entrepreneurship in nursing: an integrative literature review. Rev Bras Enferm. 2019;72(1):301-10.

6. Colichi RM, Lima SA. Empreendedorismo na enfermagem: comparação com outras profissões da saúde. Rev Eletr Enf. 2018;20(20):1-11.

7. Muraro R, Lazzari F, Eberle L, Milan GS, Verruck F. Evaluation of entrepreneur profile in academic environment. Rev Gestão Desenvolv. 2018;15(2):136-56.

8. Corrêa AK, Prebill GM, Ruiz JC, Souza MC, Santos RA. First-years student profile in the "bachellor's degree with a teaching credential in nursing" program at a Brazilian public university. Educ Rev. 2018;34 e185913:1-34.
9. Carleto CT, Moura RC, Santos VS, Pedrosa LA. Adaptation to university and common mental disorders in nursing undergraduate student. Rev Eletr Enf. 2018;20(1):1-10.

10. Mussi FC, Pires CG, Carneiro LS, Costa AL, Ribeiro FM, Santos AF. Comparison of stress in freshman and senior nursing students. Rev Esc Enferm USP. 2019;53:e03431.

11. Silva RM, Costa AL, Mussi FC, Lopes VC, Batista KM, Santos OP. Health alterations in nursing students after a year from admission to the undergraduate course. Rev Esc Enferm USP. 2019;53:e03450.

12. Melo RC, Queirós PJ, Tanaka LH, Costa PJ, Bogalho Cl, Oliveira PI. Undergraduate nursing students' difficulties during clinical training: perception of the main causes. Rev Enferm Ref. 2017;4(15):55-62.

13. Gleriano JS, Marca NC, Justi J. Profile and meaning for training in nursing. Rev Saúde Desenvolv. 2017;11(6):84-101.

14. Geovanini T, Moreira A, Machado WC. História da enfermagem: versões e interpretações. Rio de Janeiro: Revinter; 2018.

15. Latreille AC, Machado Sobrinho S, Warmling AM, Ribeiro DM, Amante CJ. Perfil socioeconômico dos graduandos em Odontologia da Universidade Federal de Santa Catarina. Rev ABENO. 2015;15(1):86-96.

16. Valente GS, Silva AC, Valente GL. Entrepreneurship as a tool for the nurse's work. Rev Enferm UFPE On line. 2017;11(4)1595-602.

17. Figueiredo MA, Peres MA. The identity of the female nurse: a reflection from the perspective of Dubar. Rev Enferm Ref. 2019;(20):149-54.

18. Eminoglu A, Gungormus Z. Entrepreneurial characteristics and inclinations of nursing students. Int J Caring Sci. 2019;12(2):684-98.

19. Richter SA, Santos EP, Kaiser DE, Capellari C, Ferreira GE. Being an entrepreneur in nursing: challenges to nurses in a strategic leadership position. Acta Paul Enferm. 2019;32(1):46-52.

20. Crevacore CA, Duffield CM, Twigg DE. Undergraduate registered nursing students working as assistants in nursing within the acute care environment: program development and discussion. Collegian. 2019;26(2):256-61.

21. Ferreira AM, Rossaneis MA, Oliveira JL, Haddad MC, Vannuchi MT. The entrepreneur profile of nursing residents. Rev Baiana Enferm. 2018;32:e27365

22. Solesvik MZ. Entrepreneurial competencies and intentions: The role of higher education. Forum Scientiae Oeconomia. 2019;7(1):9-23.

23. Costa RR, Medeiros SM, Coutinho VR, Mazzo A, Araújo MS. Satisfaction and self-confidence in the learning of nursing students: randomized clinical trial. Esc Anna Nery. 2020;24(1):e20190094.

24. Kajander-Unkuri S, Meretoja R, Katajisto J, Leino-Kilpi H, SuikkalaA. Students' self-assessed competence levels during nursing education continuum - a cross-sectional survey. Int J Nurs Educ Scholarsh. 2020;17(1):/j/ ijnes.2020.17.issue-1/ijnes-2019-0050/jines-2019-0050.xml.

25. Caveião C, Peres AM, Zagonel IP, Amestoy SC, Meier MJ. Teachinglearning tendencies and strategies used in the leadership development of nurses. Rev Bras Enferm. 2018;71(4 Suppl 4):1531-9. 